\title{
POWER OF A CLASS OF GOODNESS-OF-FIT TESTS I
}

\author{
Christopher S. Withers ${ }^{1}$ And Saralees Nadarajah ${ }^{2}$
}

\begin{abstract}
Consider testing whether $F=F_{0}$ for a continuous cdf on $R=(-\infty, \infty)$ and for a random sample $X_{1}, \ldots, X_{n}$ from $F$. We derive expansions of the associated asymptotic power based on the Cramer-von Mises, Kolmogorov-Smirnov and Kuiper statistics. We provide numerical illustrations using a double-exponential example with a shifted alternative.
\end{abstract}

Mathematics Subject Classification. 62F03; 62F05; 62F12.

Received February 11, 2007. Revised September 24, 2007 and February 25, 2008.

\section{INTRODUCTION}

The two main purposes of goodness-of-fit tests seem to be (a) as a preliminary test to see if the distribution underlying the data is close to a particular distribution so that tests designed for that distribution can then be applied (for example, the exponential distribution is usually assumed in reliability) or (b) in testing to see if there has been a change in the distribution underlying the data (accumulated data may have been used to estimate the distribution underlying the data).

Traditional criteria governing the choice of the goodness-of-fit test to be used (i.e. power and efficiency) have been almost unavailable (a) because the theory has been largely restricted to simple or parametric alternatives and (b) because the theoretical tools needed have not been available.

This paper is the first part of an attempt to synthesize ideas of Hoadley, Abrahamson, Bahadur, Chernoff, Hodges and Lehmann, and others with the methods of the calculus of variations, and differential and integral equations, in order to develop the theory of, and compute the efficiencies of, a wide range of goodness-of-fit tests when the underlying distribution is continuous and univariate. Several authors have attempted to develop this theory, but their treatments have not been comprehensive. In the sequence of papers, we aim to provide a comprehensive treatment of the problem.

Some recent work on the subject are as follows. [14] studied the asymptotic power (AP) of the two-sided one-sample Kolmogorov-Smirnov test by computing a second-order expansion of its AP function. The study explains to a large extent how the Kolmogorov-Smirnov test distributes its power in the space of all alternatives. [24] showed that the second derivative of the AP function can be employed to obtain global upper bounds for the efficiency. The bounds were shown to be least in the class of tests with a convex and centrally symmetric acceptance region. [8] generalized earlier results of [14] for Kolmogorov-Smirnov and related tests. As a

\footnotetext{
Keywords and phrases. Asymptotic power, Brownian bridge, goodness-of-fit, Pitman efficiency.

1 Applied Mathematics Group, Industrial Research Limited, Lower Hutt, New Zealand.

2 School of Mathematics, University of Manchester, Manchester M60 1QD, UK; mbbssn@manchester.ac.uk
} 
sample application, the local asymptotic relative efficiency of the two-sided Kolmogorov-Smirnov test of a simple hypothesis was considered. [9] investigated asymptotic and finite-sample-size properties of power functions, showing that the power of any non-parametric test is "flat" except for directions of alternatives coming from a finite-dimensional subspace which is typical for and depends on the test under consideration. Rahnenführer [17] provided a further extension of the results due to [8]. For other recent work on this area, see Section 14.6 of [13].

The following notation will be needed: let $F_{n}$ denote the empirical cdf of a random sample $X_{1}, \ldots, X_{n}$ from a distribution function $F$. Let $\psi$ denote a non-negative function on $[0,1]$. Define

$$
\begin{aligned}
T_{n m}(\psi) & =\left\|F_{n}-F_{0} \mid \psi\left(F_{0}\right)\right\|_{F_{0}, m}, \quad 0 \leq m \leq \infty \\
T_{n m}^{+}(\psi) & =\left\|\left(F_{n}-F_{0}\right) \psi\left(F_{0}\right)\right\|_{F_{0}, m}, \quad m=1,3,5, \ldots \\
D_{n}(\psi) & =\left\|F_{n}-F_{0} \mid \psi\left(F_{0}\right)\right\|_{F_{0}, \infty}, \\
D_{n}^{+}(\psi) & =\sup \left(F_{n}-F_{0}\right) \psi\left(F_{0}\right), \\
D_{n}^{-}(\psi) & =\sup \left(F_{0}-F_{n}\right) \psi\left(F_{0}\right), \\
V_{n}(\psi) & =D_{n}^{+}(\psi)+D_{n}^{-}(\psi),
\end{aligned}
$$

where

$$
\|G\|_{F, m}=\left\{\begin{array}{lll}
\left(\int G^{m} d F\right)^{1 / m}, & \text { if } & 0<m<\infty \\
\sup G, & \text { if } & G \geq 0, m=\infty .
\end{array}\right.
$$

Further, let $\|G\|_{m}=\|G\|_{U, m}$, where $U(\cdot)$ is the cdf of a Uniform $(0,1)$ random variable.

Let $X_{1}, \ldots, X_{n}$ be a random sample from $F$ a continuous cdf on $R=(-\infty, \infty)$. We wish to test whether $F=F_{0}$, a given continuous cdf on $R$. As candidates we consider $T_{n 2}(\psi)$ and $V_{n}(\psi)$ defined by (1.1) and (1.2), respectively. This class consists of the Cramer-von Mises, Kolmogorov-Smirnov and Kuiper statistics, $T_{n 2}(1), T_{n \infty}$ (1) and $V_{n}(1)$ whose asymptotic null distributions are given in $[3,12]$ and $[21]^{1}$.

In this paper, we show how the $\mathrm{AP}$ of $T_{n 2}(\psi)$ may be computed. We compare it with the $\mathrm{AP}$ of $T_{n 2}(1)$, $D_{n}(1), V_{n}(1)$ to the envelope power function for a particular example, the double-exponential shift family. This example has the score function leading to the canonical gradient of the median functional, see Example 2.3 of [10]. It should be emphasized that the comparisons being considered are only local in nature. The proofs of all results are given in Section 3.

\section{Asymptotic POWER}

Consider a sequence of problems in which one tests $F=F_{0}$ against $F=F_{1}$ where the alternative varies with $n$, say $F_{1}=H_{n}$. If $H_{n} \rightarrow F_{0}$ sufficiently rapidly, then the AP of a fixed $\alpha$ level test will tend to a number less than one. We shall assume

$$
H_{n}\left(F_{0}^{-1}(u)\right)=u+n^{-1 / 2} r(u)+o\left(n^{-1 / 2}\right)
$$

uniformly in $u \in[0,1]$, where $r(u)$ is bounded. For example, if $H_{n}(x)=F\left(x, \theta n^{-1 / 2}\right)$ and there is a $K$ such that for all $x$, and all $a$ in $[0, \theta]$

$$
|\partial F(x, a) / \partial a|<K, \quad\left|\partial^{2} F(x, a) / \partial a^{2}\right|<K
$$

where $F(x, 0)=F_{0}(x)$, then (2.1) holds with $r(u)=\theta p(u)$, where

$$
p(u)=\left[\partial F\left(F_{0}^{-1}(u), \theta\right) / \partial \theta\right]_{\theta=0} .
$$

\footnotetext{
${ }^{1}$ Their percentiles are conveniently given for all $n$ in a table of [15].
} 
Alternatives satisfying (2.1) are known as contiguous alternatives. Let $y(\cdot)$ be a Brownian bridge on $[0,1]$, i.e. a Gaussian process such that $E y(s)=0$ and $E y(s) y(t)=s-s t$ for $0 \leq s \leq t \leq 1$. Then it is well known (cf. [16]) that

$$
\left\{n^{1 / 2}\left(F_{n}\left(H_{n}^{-1}\left(u_{i}\right)\right)-u_{i}\right)\right\} \stackrel{\mathcal{L}}{\rightarrow}\left\{y\left(u_{i}\right)\right\}
$$

for $0 \leq u_{i} \leq 1$. Here, $X_{n} \stackrel{\mathcal{L}}{\rightarrow} X$ means that $P\left(X_{n} \leq x\right) \rightarrow P(X \leq x)$ as $n \rightarrow \infty$ for $P(X \leq x)$ continuous at $x$.

The techniques of [3] are extended to prove the following theorems. Theorems 2.1, 2.2 and part of Theorem 2.3 are known results, see Chapter 5 of [19]. Theorems 2.4 to 2.7 provide expansions of the local AP for some classical goodness-of-fit statistics. For instance, Note 2.4 expands the AP for the $D_{n}^{+}(1)$ statistic. Theorem 2.5 expands the AP for the $D_{n}(1)$ statistic. Finally, Theorem 2.6 expands the AP for the $V_{n}(1)$ statistic. These results do not appear to have been known before in the detail given. Several examples are used to illustrate the results for the double-exponential shift family. Among other things, the examples are used to compare the AP of the goodness-of-fit statistics with an approximate AP given by a Gaussian power function.

Theorem 2.1. Suppose $F=H_{n}$ satisfies (2.1). Suppose $r \psi$ has finite $L_{p}(0,1)$ norm. Then

$$
\begin{array}{rlll}
n^{1 / 2} T_{n m}(\psi) & \stackrel{\mathcal{L}}{\rightarrow} & \||r+y| \psi\|_{m}, & 0<m \leq \infty, \\
n^{1 / 2} T_{n m}^{+}(\psi) & \stackrel{\mathcal{L}}{\rightarrow} & \|(r+y) \psi\|_{m}, & m=1,3,5, \ldots, \\
n^{1 / 2} D_{n}^{+}(\psi) & \stackrel{\mathcal{L}}{\rightarrow} & \sup (r+y) \psi, \\
n^{1 / 2} D_{n}^{-}(\psi) & \stackrel{\mathcal{L}}{\rightarrow} & \sup -(r+y) \psi, \\
n^{1 / 2} V_{n} & \stackrel{\mathcal{L}}{\rightarrow} & \sup (r+y) \cdot \psi+\sup -(r+y) \cdot \psi,
\end{array}
$$

as $n \rightarrow \infty$.

Note 2.1. [16] proved Theorem 2.1 for $T_{n \cdot \infty}$ (1), $D_{n}^{+}(1)$ and gave bounds on the power functions for the sequence of composite alternatives.

Theorem 2.2. Suppose $r \psi$ has finite $L_{p}(0,1)$ norm. Let $g(u)=\left[2\left(u-u^{2}\right) \ln \ln \left(u-u^{2}\right)^{-1}\right]^{1 / 2}$.

(i) $\|g \psi\|_{m \vee 1}<\infty,\||r| \psi\|_{m \vee 1}<\infty \Rightarrow\|(r+y) . \psi\|_{k}$ exists almost surely for $k=1,3,5, \ldots, K \leq m$, and ||$|r+y| \cdot \psi \|_{k}$ exists almost surely for $0<k \leq m$, where $s \vee t=\max (s, t)$.

(ii) If $\sup _{(0,1 / 2)} g \cdot \psi=\infty$ and if for some $\epsilon>0$,

$$
(0, \epsilon) \subset\left\{\begin{array}{llll}
\{r \geq 0\}, & \text { then } & \sup (r+y) \psi=\infty & \text { almost surely, } \\
\{r \leq 0\}, & \text { then } & \sup -(r+y) \psi=\infty & \text { almost surely }
\end{array}\right.
$$

If $\sup _{(1 / 2,1)} g \cdot \psi=\infty$ and if for some $\epsilon>0$,

$$
(1-\epsilon, 1) \subset\left\{\begin{array}{lll}
\{r \geq 0\}, & \text { then } \sup (r+y) \psi=\infty & \text { almost surely, } \\
\{r \leq 0\}, & \text { then } \sup -(r+y) \psi=\infty & \text { almost surely. }
\end{array}\right.
$$

Theorem 2.3. Let

$$
W_{2}=\int_{0}^{1}(r+y)^{2} \psi^{2}, B=r \psi
$$

We have (a):

$$
E e^{-t W_{2}}=\prod_{j=1}^{\infty}\left(1+2 t / \lambda_{j}\right)^{-1 / 2} \mathrm{e}^{-S(t)}
$$


and (b):

$$
\begin{aligned}
E W_{2} & =\int_{0}^{1}\left[B(x)^{2}+K_{1}(x, x)\right] \mathrm{d} x \\
\operatorname{var} W_{2} & =2 \int_{0}^{1} \int_{0}^{1}\left[2 B(x) B(z)+K_{1}(x, z)\right] K_{1}(x, z) \mathrm{d} x \mathrm{~d} z,
\end{aligned}
$$

where

$$
\begin{aligned}
S(t) & =t \sum_{1}^{\infty} b_{j}^{2}\left(1+2 t / \lambda_{j}\right)^{-1} \\
& =t \int_{0}^{1} B^{2}-2 t^{2} \int_{0}^{1} \int_{0}^{1} B(x) B(z) K(x, z:-2 t) \mathrm{d} x \mathrm{~d} z, \\
b_{j} & =\int_{0}^{1} B f_{j}, \\
K_{k}(x, z) & =\sum_{j=1}^{\infty} \lambda_{j}^{-k} f_{j}(x) f_{j}(z)=\int_{0}^{1} K_{k-1}(x, u) K_{1}(u, z) \mathrm{d} u,
\end{aligned}
$$

$\left\{\lambda_{j}, f_{j}\right\}$ are the eigenvalues and eigenfunctions of $K_{1}(x, z)=(\min (x, z)-x z) \psi(x) \psi(z), K(x, z: \lambda)$ is the "resolvent kernel" $\sum_{k=1}^{\infty} \lambda^{k-1} K_{k}(x, z)$ (see [4], p. 141, for alternative expressions), $\{y \psi\} \stackrel{\mathcal{L}}{=}\left\{\sum_{1}^{\infty} Y_{i} f_{i} \lambda_{i}^{-1 / 2}\right\}$, where $Y_{i}$ are independent standard normal random variables, $K_{k}(x, z)$ is the "kth iterated kernel", $k=2,3, \ldots$ and we assume that $\psi$ satisfies either

$(E): \psi$ is continuous on $(0,1)$ and for all $\epsilon>0, \psi(t) t\left[(1-s) \psi^{\prime}(s)-\psi(s)\right]$ is continuous for $t \vee \epsilon \leq s \leq 1-\epsilon$ and $\psi(t)(1-t)\left[s \psi^{\prime}(s)+\psi(s)\right]$ is continuous for $\epsilon \leq s \leq t \vee(1-\epsilon)$; or

$(F): \psi$ is continuous in $[a, b]$ and zero elsewhere, where $0 \leq a<b \leq 1$,

see [3], p. 198.

Note 2.2. $x_{\alpha}=G^{-1}(1-\alpha)$, where $G(x)=P\left(W_{0} \leq x\right)$ and $W_{0}=\int_{0}^{1} y^{2} \psi^{2}$, is tabulated in [3] for $\psi=1$.

Example 2.1. Suppose $\psi=1$. Let $z=(2 t)^{1 / 2}$. Then

$$
\prod_{j=1}^{\infty}\left(1+2 t / \lambda_{j}\right)^{-1 / 2}=(z / \sinh z)^{1 / 2}
$$

and

$$
K(a, b:-2 t)=\frac{\sinh (b z) \cdot \sinh (z-a z)}{z \sinh z}
$$

for $1 \geq a \geq b \geq 0$.

Example 2.2. In the particular case $\psi=1, \dot{F}_{0}(x)=1 / 2 \mathrm{e}^{-|x|}$ and $H_{n}(x)=F_{0}\left(x-\theta n^{-1 / 2}\right)$ (where $\dot{F}_{0}(x)$ denotes the first derivative of $F_{0}(x)$ with respect to $\left.x\right)$ the $A P$ of $T_{n 2}(1)$ is

$$
P\left(W_{2}>x_{\alpha}\right)=1-2^{3 / 2} \mathrm{e}^{-\theta^{2} / 2} \sum_{n=0}^{\infty} \frac{\theta^{2 n}}{n !} \sum_{k=0}^{\infty} 2^{-n / 2-3 / 4} a_{k n} R_{n}\left(A_{k}, x_{\alpha}\right),
$$


where

$$
\begin{aligned}
a_{k n}= & \sum_{i=0}^{k}\left(\begin{array}{c}
n-1 / 2 \\
i
\end{array}\right)\left(\begin{array}{c}
-n-1 / 2 \\
k-i
\end{array}\right)(-1)^{i} \\
A_{k}= & (k+1 / 2) 2^{1 / 2} \\
R_{n}(A, x)= & A \pi^{-1} x^{n / 2-3 / 4} 2^{-5 / 4-n / 2} \frac{\Gamma(3 / 4-n / 2)}{\Gamma(3 / 4+n / 2)} \mathrm{e}^{-c} \\
& \sum_{i=0}^{n} \frac{n !}{(n-i) !} \sum_{k=0}^{[i / 2]} \frac{(-1)^{i+k+n}}{(i-2 k) ! k !} 2^{-k} c^{n / 2-k-1 / 4} K_{1 / 4+i-k-n / 2}(c),
\end{aligned}
$$

where $c=A^{2} / 8 x$ and $K_{a}(c)$ is the modified Bessel function (e.g. [5], p. 5) and $x_{\alpha}=G^{-1}(1-\alpha)$ is tabulated in Table 1: [3], p. 203.

Theorem 2.4. (Local AP)

(i) If $H_{n}(x)=F_{0}\left(x-\theta n^{-1 / 2}\right), x_{\alpha}=G^{-1}(1-\alpha), G(x)=P\left(W_{0} \leq x\right), W_{0}=\int_{0}^{1} y^{2} \psi^{2}$ and $b=\psi p$ with $p$ defined by (2.2) then as $\theta \rightarrow 0$

$$
P\left(W_{2}>x_{\alpha}\right)=\alpha+\theta^{2} A\left(x_{\alpha}\right)+o\left(\theta^{2}\right),
$$

where

$$
A(x)=\dot{G}(x) \int_{0}^{1} b^{2}+\sum_{k=1}^{\infty}(-2)^{k} G^{(k+1)}(x) \int_{0}^{1} b(u) \mathrm{d} u \int_{0}^{1} b(v) K_{k}(u, v) \mathrm{d} v
$$

where $K_{k}(u, v)$ is given by (2.3) and $G^{(k)}(x)$ denotes the kth derivative of $G(x)$ with respect to $x$.

(ii) In the particular case $\psi=1$ (i) is true and

$$
A(x)=\dot{G}(x) \int_{0}^{1} b^{2}-2 \int_{0}^{1} b(u) \int_{0}^{u} b(v) S(x, u, v) \mathrm{d} v
$$

where

$$
\begin{aligned}
S(x, 1-u, v)= & -\sum_{j=1}^{4} d_{j} \sum_{i=0}^{\infty}\left(\begin{array}{c}
-3 \\
i
\end{array}\right)(-1)^{i} c_{i j}(u, v) \pi^{-1} x^{-9 / 4} f\left(c_{i j}(u, v)^{2} / 8 x\right), \\
f(c)= & \mathrm{e}^{-c}\left[c^{5 / 4} K_{5 / 4}(c)+3(c-1) c^{1 / 4} K_{1 / 4}(c)+3\left(c^{2}-1\right) c^{-3 / 4} K_{3 / 4}(c)\right. \\
& \left.\left.+c^{5 / 4} K_{7 / 4} c\right)\right], \\
d_{1}= & d_{4}=-d_{2}=-d_{3}=1, \\
c_{i j}(u, v)= & (3 / 2-u-v+2 i) \cdot 2^{1 / 2}+2^{3 / 2} \begin{cases}0, & j=1, \\
u, & j=2, \\
v, & j=3, \\
u+v, & j=4 .\end{cases}
\end{aligned}
$$

Example 2.3. In the particular case $\psi=1$ and $\dot{F}_{0}(x)=1 / 2 \mathrm{e}^{-|x|}$ Theorem 2.4(i) is true and

$$
A\left(x_{\alpha}\right)=1 / 2(1-\alpha)-2^{-3 / 4} \sum_{k=0}^{\infty} a_{k 1} \Omega\left(A_{k}, x_{\alpha}\right),
$$


where (2.4) and (2.5) give $a_{k 1}$ and $A_{k}$, respectively, and

$$
\Omega(A, x)=\left(\frac{A^{3}}{2 x}\right)^{1 / 2} \pi^{-1} \cdot \exp \left\{-\frac{A^{2}}{8 x}\right\} \cdot\left[K_{3 / 4}\left(\frac{A^{2}}{8 x}\right)-K_{1 / 4}\left(\frac{A^{2}}{8 x}\right)\right] .
$$

Examples 2.3 and 2.4 have been used to compare the AP and the "approximate AP", defined to be

$$
\Phi\left(-z_{\alpha / 2}+I^{1 / 2} e(\alpha)^{1 / 2} \theta\right)+\Phi\left(-z_{\alpha / 2}-I^{1 / 2} e(\alpha)^{1 / 2} \theta\right)
$$

where $I$ is given by (2.8) below, $e(\alpha)=A\left(x_{\alpha}\right) / z_{\alpha / 2} \phi\left(z_{\alpha / 2}\right)$ for the double-exponential case above, $z_{\alpha}=\Phi^{-1}(1-$ $\alpha)$, and $\phi(\cdot)$ and $\Phi(\cdot)$ are the pdf and the cdf of the standard normal distribution. See Figures 1-3.

Note 2.3. This method extends to approximating the AP by a polynomial in $\theta$ arbitrarily well but with the number of integrals increasing with the degree of the polynomial; it also extends to $\psi$ which are piecewise of the form $\psi(t)=1 /(a+b t)$; when $\psi=1$ it extends to give the AP for $H_{n}$ such that $p(x)$ is piecewise of the form $a+b x$ (e.g. the double-exponential example for which the AP of $D_{n}^{+}$(1) was found on p. 272 of [7].)

In the remainder of this section, we assume $H_{n}(x)=F\left(x, \theta n^{-1 / 2}\right)$. We now give some generalizations of VI.4.5, p. 230 of [7] and [2] which gave the local AP of $D_{n}^{+}(1)$ and $D_{n}^{+}\left(\psi_{1}\right)$ when $F(x, \theta)=F_{0}(x-\theta)$ and

$$
\psi_{1}(t)= \begin{cases}1 / t, & \text { in }[a, 1] \\ 0, & \text { in }[0, a)\end{cases}
$$

Define the (fixed $\alpha$ ) asymptotic efficiency, $e(\alpha, \theta)=\lim _{n \rightarrow \infty} n_{2} / n$, where $n_{2}$ is the sample size required by the most powerful test of $\theta=0$ versus $\theta>0$ in the one-sided case, or versus $\theta \neq 0$ in the two-sided case, in order to achieve the power that the test statistic has with a sample of $n$.

Let $\theta_{2}=\left(n_{2} / n\right)^{1 / 2} \theta$. The most powerful test of $F(x, 0)$ versus $F\left(x, \theta n^{-1 / 2}\right)=F\left(x, \theta_{2} n_{2}^{-1 / 2}\right)$ is the simple likelihood ratio (LR) test which has the AP

$$
\Phi\left(I^{1 / 2} \theta-z_{\alpha}\right)=\alpha+I^{1 / 2} \theta \phi\left(z_{\alpha}\right)+o(\theta)
$$

In the two-sided case asymptotically the composite LR test (and the test based on $\left|\sum_{i=1}^{n} \dot{p}\left(F_{0}\left(X_{i}\right)\right)\right|$ ) are locally most powerful with the AP

$$
\Phi\left(I^{1 / 2} \theta-z_{\alpha / 2}\right)+\Phi\left(-I^{1 / 2} \theta-z_{\alpha / 2}\right)=\alpha+I \theta^{2} z_{\alpha / 2} \phi\left(z_{\alpha / 2}\right)+o\left(\theta^{2}\right),
$$

where

$$
I=\int_{0}^{1} \dot{p}^{2}
$$

the Fisher information. Along the lines of [7], p. 273, this suggests approximating the AP on the one-sided case by $\Phi\left(e(\alpha)^{1 / 2} I^{1 / 2} \theta-z_{\alpha}\right)$, where

$$
e(\alpha)=\lim _{\theta \rightarrow 0} e(\alpha, \theta)
$$

the local asymptotic efficiency, since if $\theta_{2}=e(\alpha)^{1 / 2} \theta$ then $F\left(x, \theta_{2} n_{2}^{-1 / 2}\right) \approx F\left(x, \theta n^{-1 / 2}\right)$.

Note 2.4. For $D_{n}^{+}(1), x_{\alpha}=\left(-2^{-1} \ln \alpha\right)^{1 / 2}$. [2] and [7] (p. 275) have given its AP. It satisfies (i)

$$
P\left(\sup (y+\theta p) \geq x_{\alpha}\right)=\alpha-2 \alpha x_{\alpha} \theta \int_{0}^{1} \dot{p}(u) \Lambda(\alpha, u) \mathrm{d} u+o(\theta),
$$


Cramer-von Mises Statistic

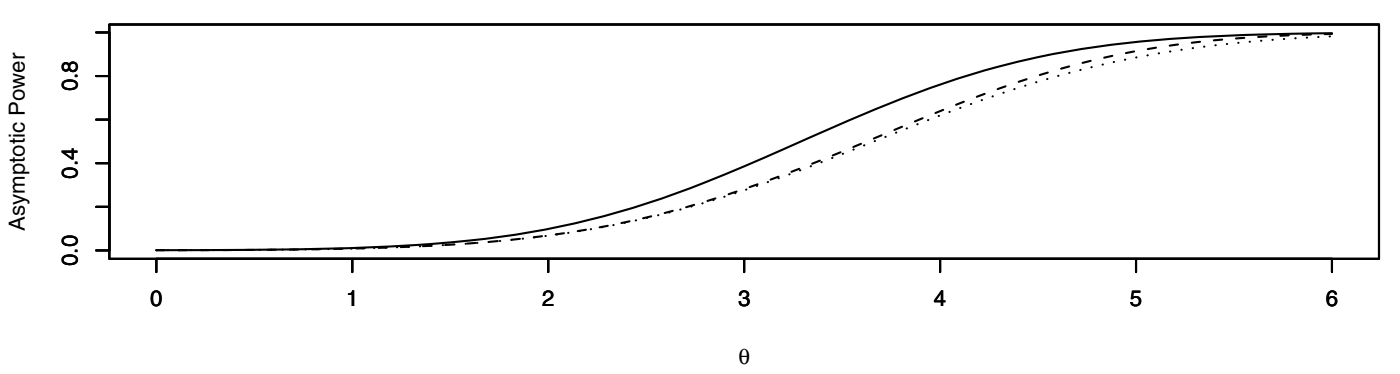

Kolmogorov-Smirnov Statistic

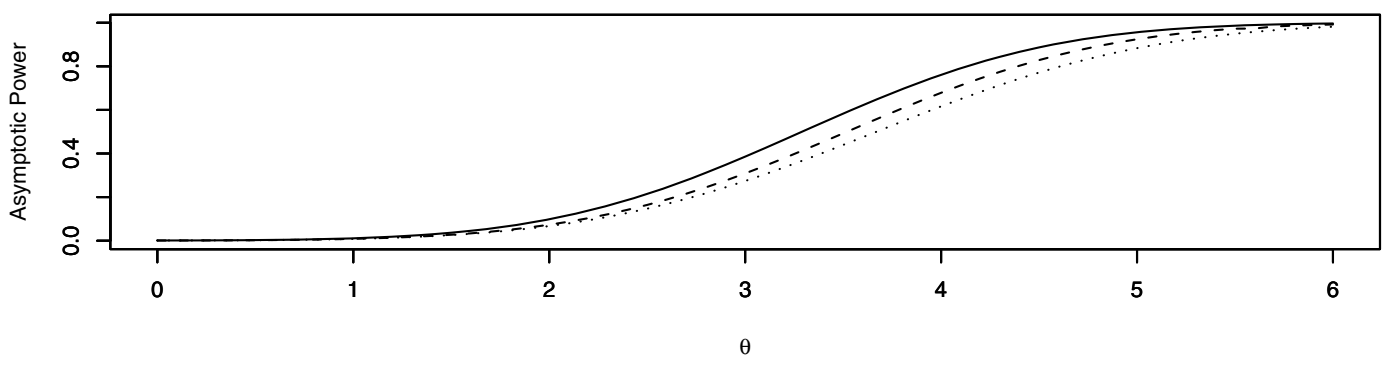

Kuiper Statistic

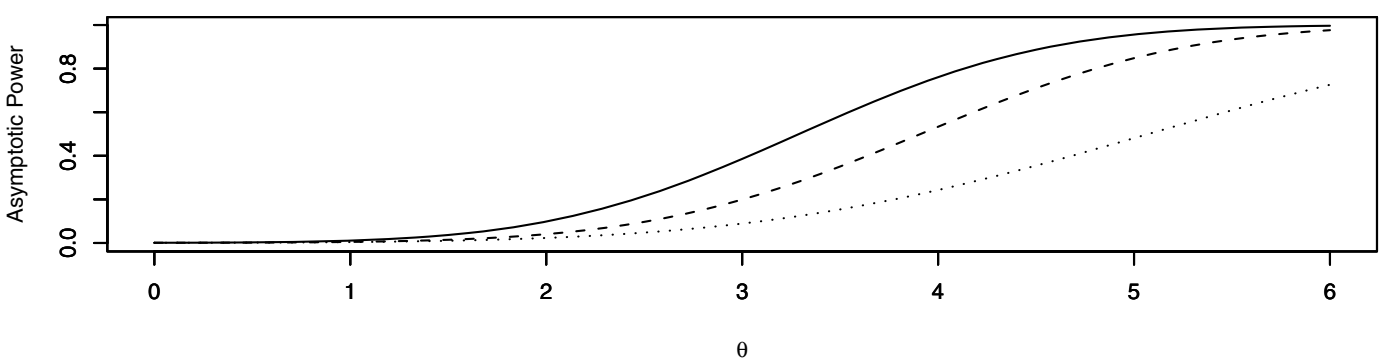

Figure 1. AP for the double-exponential with shift alternative using $0.1 \%$ level: the solid curve shows the AP from the LR test; the curve of dashes shows the AP using the specified statistic; and, the curve of dots shows the approximate AP using the specified statistic.

where

$$
\Lambda(\alpha, u)=2 \Phi\left(x_{\alpha}(2 u-1)\left(u-u^{2}\right)^{-1 / 2}\right)-1
$$

and (ii) $e(\alpha)=4 \pi \alpha^{2} \ln \alpha^{-1} \mathrm{e}^{z_{\alpha}^{2}}\left(\int_{0}^{1} \dot{p}(u) \Lambda(\alpha, u) \mathrm{d} u\right)^{2} / \int_{0}^{1} \dot{p}^{2}, \lim _{\alpha \rightarrow 0} e(\alpha)=4 p(1 / 2)^{2} / \int_{0}^{1} \dot{p}^{2}$.

Note 2.5. It can be shown that the Pitman efficiency of $D_{1}^{+}(1)$ is $4(\sup p)^{2} / \int_{0}^{1} \dot{p}^{2}$. Hence, Hajek's local efficiency or its limit as $\alpha \rightarrow 0$ need not equal the Pitman efficiency. 


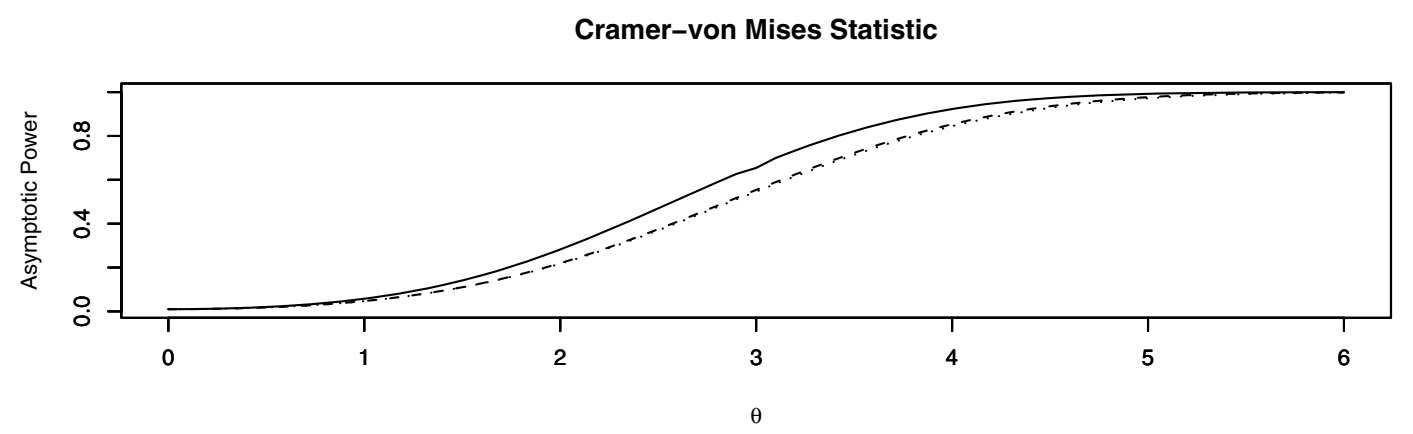

Kolmogorov-Smirnov Statistic

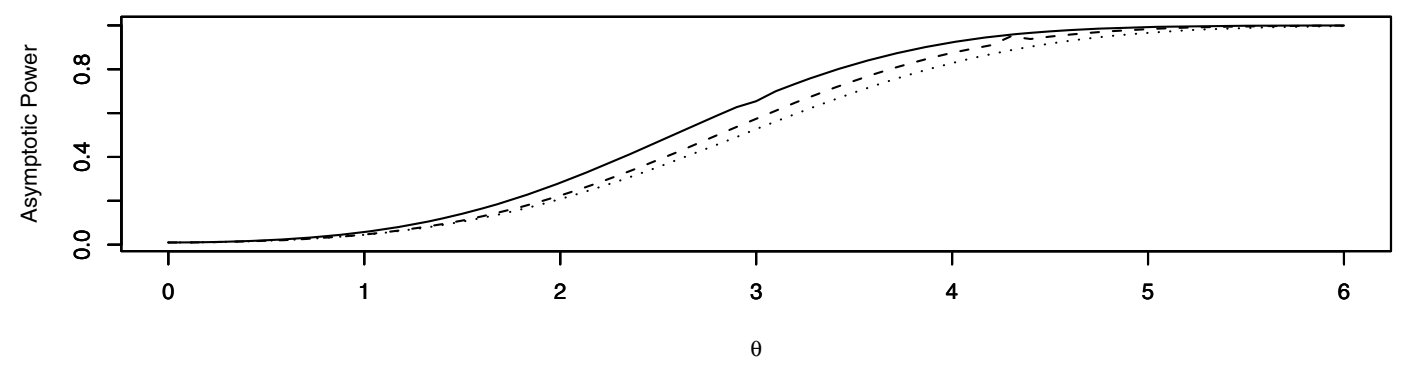

Kuiper Statistic

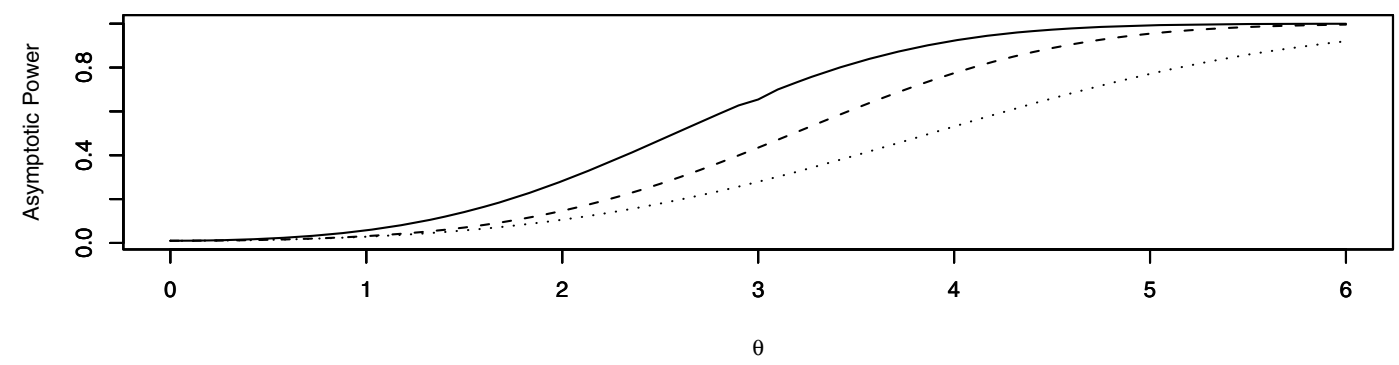

Figure 2. AP for the double-exponential with shift alternative using $1 \%$ level: the solid curve shows the AP from the LR test; the curve of dashes shows the AP using the specified statistic; and, the curve of dots shows the approximate AP using the specified statistic.

Theorem 2.5. Let $G(x)=P\{\sup |y| \leq x\}, \lambda=G^{-1}(1-\alpha)$. The AP of $D_{n}(1)$ is

$$
\begin{aligned}
P(\sup |y+\theta p| \geq \lambda)= & \alpha+\theta^{2} / 2(1-\alpha) \int_{0}^{1} \dot{p}^{2}-2 \int_{0}^{1} \ddot{p}(u) \mathrm{d} u \int_{u}^{1} p(v) \mathrm{d} v \\
& \int_{-\lambda}^{\lambda} x \mathrm{~d} x \int_{-\lambda}^{\lambda} z \mathrm{~d} z Q(x, z, u, v)+o\left(\theta^{2}\right)
\end{aligned}
$$


Cramer-von Mises Statistic

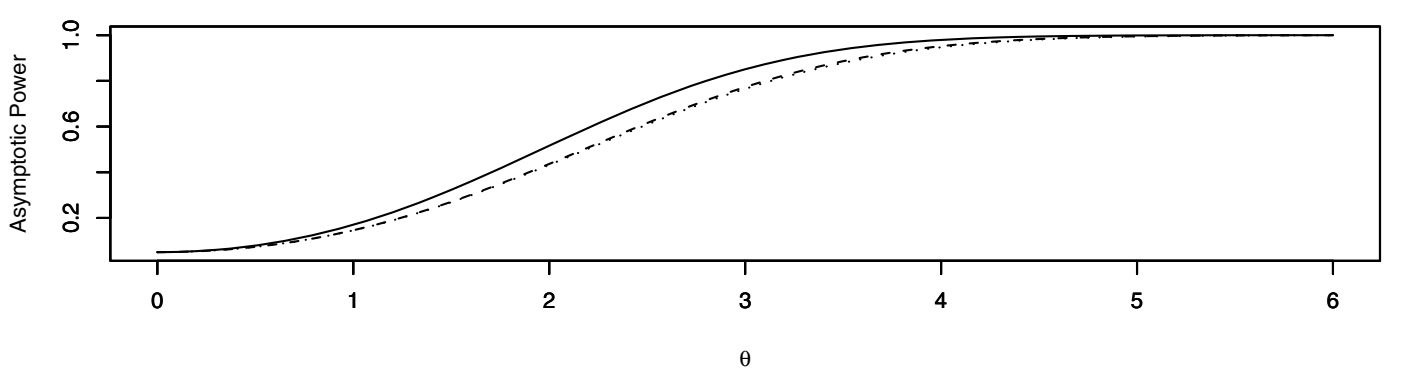

Kolmogorov-Smirnov Statistic

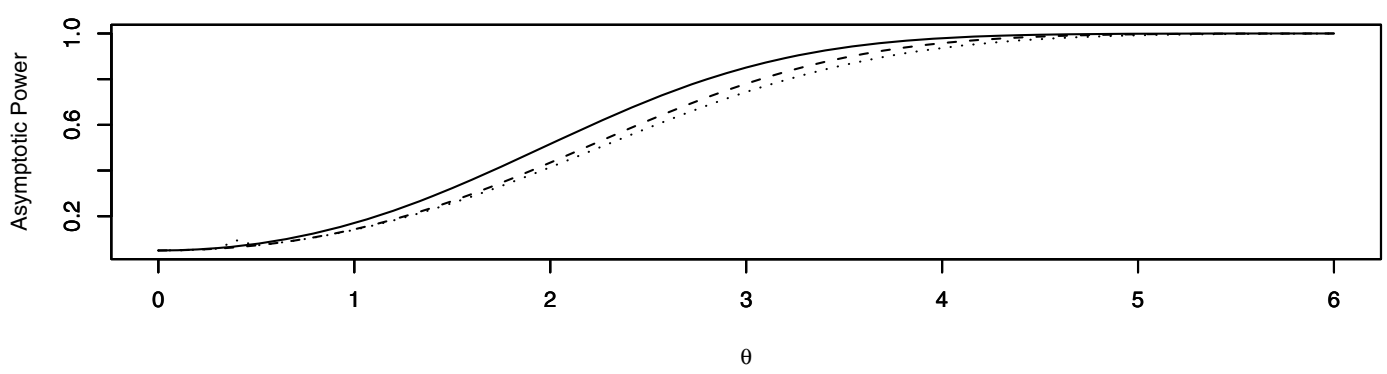

Kuiper Statistic

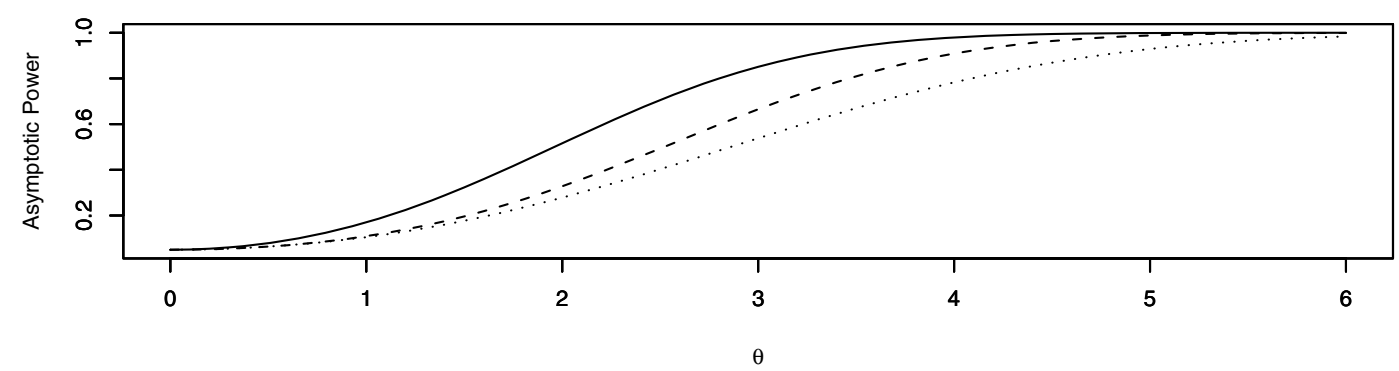

Figure 3. AP for the double-exponential with shift alternative using $5 \%$ level: the solid curve shows the AP from the LR test; the curve of dashes shows the AP using the specified statistic; and, the curve of dots shows the approximate AP using the specified statistic.

where

$$
\begin{aligned}
& Q(x, z, u, v)=[2 \pi|\Sigma|]^{-1} \exp \left[(-1 / 2)(x z) \Sigma^{-1}\left(\begin{array}{l}
x \\
z
\end{array}\right)\right] f_{u v}(x z \lambda), \\
& f_{u v}(x z \lambda)=K(u, 0, x, \lambda) K(v-u, x, z, \lambda) K(1-v, z, 0, \lambda), \\
& K(u, x, z, \lambda)=1-\sum_{m=1}^{\infty}\left[\exp \left(-2 A_{m} / u\right)+\exp \left(-2 B_{m} / u\right)-\exp \left(-2 C_{m} / u\right)-\exp \left(-2 D_{m} / u\right)\right] \text {, } \\
& A_{m}=[(2 m-1) \lambda-x][(2 m-1) \lambda-z], \\
& B_{m}=[(2 m-1) \lambda+x][(2 m-1) \lambda+z], \\
& C_{m}=[2 m \lambda-x][2 m \lambda+z]+x z, \\
& D_{m}=[2 m \lambda+x][2 m \lambda-z]+x z, \\
& \Sigma=\left(\begin{array}{ll}
u-u^{2} & u-u v \\
u-u v & v-v^{2}
\end{array}\right)
\end{aligned}
$$


Note that $\ddot{p}(u)$ denotes the second derivative of $p(u)$ with respect to $u$.

Theorem 2.6. Set $G(x)=P\{\sup y+\sup -y \leq x\}, \lambda=G^{-1}(1-\alpha)$. The AP of $V_{n}(1)$ is

$$
\begin{aligned}
& P(\sup (y+\theta p)+\sup -(y+\theta p) \geq \lambda)= \\
& \alpha+\left(\theta^{2} / 2\right)\left[(1-\alpha) \int_{0}^{1} \dot{p}^{2}-2 \int_{0}^{1} \ddot{p}(u) \mathrm{d} u \int_{v}^{1} \ddot{p}(u) \mathrm{d} v \int x \mathrm{~d} x \int z \mathrm{~d} z Q(x, z, u, v)\right]+o\left(\theta^{2}\right),
\end{aligned}
$$

where $Q$ is defined by (2.9) in terms of

$$
\begin{aligned}
f_{u v}(x z \lambda)= & \left(\partial^{2} / \partial x \partial z\right) \iiint \iiint_{\substack{x_{i}+z_{j} \leq \lambda \\
i, j=1,3}} H\left(u, 0, x, x_{1}, z_{1}\right) \\
& H\left(v-u, x, z, x_{2}, z_{2}\right) H\left(1-v, z, 1, x_{3}, z_{3}\right) \prod_{i=1}^{3}\left(\mathrm{~d} x_{i} \mathrm{~d} z_{i}\right), \\
H(u, x, z, A, B)= & \left(8 / u^{2}\right) \sum_{m=1}^{\infty}\left[m^{2}\left(\exp \left(-2 C_{m} / u\right)+\exp \left(-2 D_{m} / u\right)\right)\right. \\
& \left.-\left(m^{2}-m\right)\left\{\exp \left(-2 A_{m} / u\right)+\exp \left(-2 B_{m} / u\right)\right\}\right],
\end{aligned}
$$

where $A_{m}, B_{m}, C_{m}, D_{m}$ are given by [2] and [7] (Pb. 15, p. 199) with $a=B+x, b=B+z, \alpha=A-x, \beta=A-z$.

Example 2.4. Suppose $H_{n}(x)=F_{0}\left(x-\theta n^{-1 / 2}\right)$ and $\dot{F}_{0}(x)=(1 / 2) \mathrm{e}^{-|x|}$. Then

(i) the $A P$ of $D_{n}(1)$ is

$$
1-\int_{-\mu}^{\mu} H(x, \mu)^{2} \mathrm{~d} \Phi\left(2^{1 / 2} x+\theta\right)
$$

where

$$
H(x, z)= \begin{cases}0, & \text { if }|x| \geq z \\ 1-2 \sum_{m=1}^{\infty}(-1)^{m+1} \exp \left(-2 m^{2} z^{2}\right) \cosh (2 m x z), & \text { if }|x|<z\end{cases}
$$

and $G(z)=H(0, z), \lambda=G^{-1}(1-\alpha), \mu=2^{1 / 2} \lambda$;

(ii) the $A P$ of $V_{n}(1)$ is

$$
1-\int_{-\mu}^{\mu} \mathrm{d} \Phi\left(2^{1 / 2} \omega+\theta\right) \int_{\max (0,-\omega)}^{\min (\mu, \mu-\omega)} M(a, a+\omega, \mu-a, \mu-a-\omega) \mathrm{d} a,
$$

where $G(x)=P\{\sup y+\sup -y \geq x\}, \lambda=G^{-1}(1-\alpha), \mu=2^{1 / 2} \lambda$,

$$
H(a, b, \alpha, \beta)=P\{-a(1-t)-b t<y(t)<\alpha(1-t)+\beta t \text { in }(0,1)\}
$$

(given by [2] and [7] (Pb. 15, p. 199)), and $M(a, b, \alpha, \beta)=2(\partial H / \partial a+\partial H / \partial b) H$.

Figures 1-3 use examples 2.2-2.4 to give the approximate AP as defined by (2.7). It appears that the AP and approximate AP are closest for the Cramer-von Mises statistic and furthest for the Kuiper statistic. The closest and furthest to the AP from the LR test are those corresponding to the Kolmogorov-Smirnov and Kuiper statistics, respectively. The exact small-sample power for the cases considered in the figures can be calculated using [20]. The small-sample power has been found for some examples by Monte Carlo methods: see [18] and $[22,23]$. For the analogs for the one-sided statistics $D_{n}^{+}(1), D_{n}^{-}(1)$, see [7], p. 274. 


\section{Proofs}

Proof of Theorem 2.3. (a) $B=\sum b_{j} f_{j},\{y \psi\} \stackrel{\mathcal{L}}{=}\left\{\sum Y_{j} \lambda_{j}^{-1 / 2} f_{j}\right\}$ imply

$$
W_{2} \stackrel{\mathcal{L}}{=} \int\left[\sum f_{j}\left(b_{j}+Y_{j} \lambda_{j}^{-1 / 2}\right)\right]^{2}=\sum\left(b_{j}+Y_{j} \lambda_{j}^{-1 / 2}\right)^{2}
$$

since $\left\{f_{j}\right\}$ are orthonormal. Set $K_{0}(x, z)=\delta(x-z)$, the Dirac delta function. Then

$$
E e^{-t W_{2}}=\prod_{j=1}^{\infty}\left(1+2 t / \lambda_{j}\right)^{-1 / 2} \cdot \mathrm{e}^{-S(t)}
$$

where

$$
\begin{aligned}
S(t) & =t \sum_{1}^{\infty} b_{j}^{2}\left(1+2 t / \lambda_{j}\right)^{-1} \\
& =t \sum_{k=0}^{\infty}(-2 t)^{k} \sum_{j=1}^{\infty} b_{j}^{2} \lambda_{j}^{-k} \\
& =t \sum_{k=0}^{\infty}(-2 t)^{k} \int_{0}^{1} \int_{0}^{1} K_{k}(x, z) B(x) B(z) \mathrm{d} x \mathrm{~d} z \\
& =t \int B^{2}-2 t^{2} \iint K(x, z:-2 t) \mathrm{d} x \mathrm{~d} z .
\end{aligned}
$$

Part (b) follows from the definition of $W_{2}$.

Proof of Example 2.1. It is shown in [3] that

$$
\prod_{j=1}^{\infty}\left(1+2 t / \lambda_{j}\right)^{-1 / 2}=\left(\frac{z}{\sinh z}\right)^{1 / 2}
$$

when $\psi=1$. Let

$$
H(x)=\left\{\begin{array}{ll}
1, & x \leq a, \\
0, & x>a,
\end{array} R(x)= \begin{cases}1, & x \leq b \\
0, & x>b\end{cases}\right.
$$

where $0<b<a<1$. Then

$$
H(x)=\sum_{1}^{\infty} h_{j} f_{j}(x)
$$

for

$$
h_{j}=\int f_{j} H=\left(2^{1 / 2} / j \pi\right)[1-\cos j \pi a]
$$

and

$$
R(x)=\sum_{1}^{\infty} r_{j} f_{j}(x)
$$

for

$$
r_{j}=\int f_{j} R=\left(2^{1 / 2} / j \pi\right)[1-\cos j \pi b] .
$$


For $k=1,2, \ldots$

$$
\begin{aligned}
\int_{0}^{a} \mathrm{~d} u \int_{0}^{b} \mathrm{~d} v K_{k}(u, v)= & \iint H(u) R(v) K_{k}(u, v) \mathrm{d} u \mathrm{~d} v \\
= & \sum_{j=1}^{\infty} h_{j} r_{j} \lambda_{j}^{-k} \\
= & (-4)^{k} 4\left[\phi_{2 k+2}(0)-\phi_{2 k+2}\left(\frac{a}{2}\right)-2 \phi_{2 k+2}\left(\frac{b}{2}\right)\right. \\
& \left.+1 / 2 \phi_{2 k+2}\left(\frac{a+b}{2}\right)+1 / 2 \phi_{2 k+2}\left(\frac{a-b}{2}\right)\right]
\end{aligned}
$$

since if $x \geq 0$

$$
2 \sum_{j=1}^{\infty} \cos (2 j \pi x) \cdot(2 \pi j)^{-2 k+2}=(-1)^{k} \phi_{2 k+2}(x)
$$

where $\phi_{n}(\cdot)$ is the $n$th Bernoulli polynomial $\left(\right.$ e.g. [11]). Differentiating both sides and noting that $\dot{\phi}_{n}(x)=$ $\phi_{n-1}(x)$ yields

$$
K_{k}(a, b)=(-4)^{k} 1 / 2\left[\phi_{2 k}\left(\frac{a+b}{2}\right)-\phi_{2 k}\left(\frac{a-b}{2}\right)\right], k=1,2, \ldots
$$

Now

$$
\sum_{n=0}^{\infty} \phi_{n}(x) t^{n}=\frac{t}{\mathrm{e}^{t}-1} \mathrm{e}^{t x}
$$

By [3],

$$
W(t)=(1+t) y\left(\frac{t}{1+t}\right)
$$

is a Wiener process and so $\phi_{2 k}(1-x)=\phi_{2 k}(x)$. Hence, $\phi_{n}(x)=B_{n}(x) / n$ ! for $B_{n}(x)$ of $[1]$, p. 806, and

$$
2 \sum_{k=0}^{\infty} t^{2 k} \phi_{2 k}(x)=\frac{t}{\mathrm{e}^{t}-1}\left(\mathrm{e}^{t x}+\mathrm{e}^{t-t x}\right)
$$

Hence,

$$
\begin{aligned}
\sum_{k=1}^{\infty}(-2 t)^{k} K_{k}(a, b) & =\sum_{k=0}^{\infty}(2 z)^{2 k} 1 / 2\left[\phi_{2 k}\left(\frac{a+b}{2}\right)-\phi_{2 k}\left(\frac{a-b}{2}\right)\right] \\
& =\frac{z}{\sinh z} \sinh (z b) \sinh (z a-z)
\end{aligned}
$$

The example is proved.

Proof of Example 2.2. For this example,

$$
r(x)= \begin{cases}-\theta x, & \text { in }[0,1 / 2] \\ -\theta(1-x), & \text { in }[1 / 2,1]\end{cases}
$$


and $S(t)$ can be shown by Theorem 2.3 (a) and Example 2.1 to equal

$$
\theta^{2}\left[\frac{1}{2}+\frac{1}{z} \cdot \frac{1-\mathrm{e}^{z}}{1+\mathrm{e}^{z}}\right]
$$

where $z=(2 t)^{1 / 2}$. Hence,

$$
\begin{aligned}
E \exp \left[-z^{2} W_{2} / 2\right] & =z^{1 / 2}(\sinh z)^{-1 / 2} \exp \left[-\theta^{2}\left(1 / 2-\frac{\tanh (z / 2)}{z}\right)\right] \\
& =2^{1 / 2} \exp \left[-\theta^{2} / 2\right] \sum_{n=0}^{\infty} \frac{\theta^{2 n}}{n !} z^{1 / 2-n} \sum_{k=0}^{\infty} a_{k n} \exp [-(k+1 / 2) z]
\end{aligned}
$$

where $a_{k n}$ are defined in the example. Using the notation

$$
\begin{aligned}
L E(x) & =\int_{0}^{\infty} E(x) \mathrm{e}^{-x t} \mathrm{~d} x, \\
L^{-1} \int_{0}^{\infty} E(x) \mathrm{e}^{-x t} \mathrm{~d} x & =E(x) \\
a(x) \otimes b(x) & =\int_{0}^{x} a(z) b(x-z) \mathrm{d} z,
\end{aligned}
$$

we have

$$
\begin{aligned}
P\left(W_{2} \leq x\right) & =L^{-1} t^{-1} E \mathrm{e}^{-t W_{2}} \\
& =2^{3 / 2} \mathrm{e}^{-\theta^{2 / 2}} \sum_{n=0}^{\infty} \frac{\theta^{2 n}}{n !} \sum_{k=0}^{\infty} a_{k n} L^{-1}\left(t^{-n / 2-3 / 4} \mathrm{e}^{-A_{k} t^{1 / 2}}\right) .
\end{aligned}
$$

Setting $c=A^{2} / 8 x, \eta=2 \theta$ for $3 / 2-2 b$ an integer,

$$
\begin{aligned}
L^{-1} \mathrm{e}^{-A t^{1 / 2}} t^{-b}= & 1 / 2 A \pi^{-1 / 2} x^{-3 / 2} \mathrm{e}^{-A^{2} / 4 x} \otimes x^{b-1} / \Gamma(b) \\
= & \frac{A x^{b-3 / 2}}{\Gamma(b)}-\pi^{-1 / 2} \int_{0}^{\infty} \exp \left[-2 c \cosh ^{2} \theta\right] \sinh ^{2 b-1} \theta \cosh ^{2-2 b} \theta \mathrm{d} \theta \\
= & \left(A 2^{-3 / 2} / \Gamma(b)\right) x^{b-3 / 2} \pi^{-1 / 2}(-1 / 2 \mathrm{~d} / \mathrm{d} c)^{3 / 2-2 b} \\
& \times\left[\mathrm{e}^{-c} \int_{0}^{\infty} \mathrm{e}^{-c(1+\cosh \eta)}(1 / 2 \sinh \eta)^{2 b-1} \mathrm{~d} \eta / 2\right] \\
= & A 2^{-3 / 2} \pi^{-1} x^{b-3 / 2}\left(-\frac{\mathrm{d}}{\mathrm{d} c}\right)^{3 / 2-2 b}\left[\mathrm{e}^{-c}(c / 2)^{-v} K_{v}(c)\right]
\end{aligned}
$$

if $b>0$, where $v=b-1 / 2$, since by p. 82 of [5], if $v>-1 / 2$

$$
\Gamma(1 / 2+v) K_{v}(c)=\pi^{1 / 2}(c / 2)^{v} \int_{0}^{\infty} \mathrm{e}^{-c \cosh t}(\sinh t)^{2 v} \mathrm{~d} t .
$$

Hence, $P\left(W_{2}>x_{\alpha}\right)$ is as given in the example, where

$$
\begin{aligned}
R_{n}(A, x) & =L^{-1} t^{-n / 2-3 / 4} \mathrm{e}^{-A t^{1 / 2}} \\
& =A 2^{-3 / 2} \pi^{-1} x^{n / 2-3 / 4}(-\mathrm{d} / \mathrm{d} c)^{-n}\left[\mathrm{e}^{-c}(c / 2)^{-v} K_{v}(c)\right]
\end{aligned}
$$


at $c=A^{2} / 8 x, v=n / 2+1 / 4$. By [5], p. 82 :

$$
\begin{aligned}
& (\mathrm{d} / \mathrm{d} c)^{-n}\left[\mathrm{e}^{-c}(c / 2)^{-v} K_{v}(c)\right]= \\
& \frac{(\pi / 2)^{1 / 2}}{\Gamma(v+1 / 2)} 2^{v}\left(\frac{\mathrm{d}}{\mathrm{d} c}\right)^{-n} \int_{0}^{\infty} \mathrm{e}^{-c t} t^{v-1 / 2}\left(1+\frac{t}{2}\right)^{v-1 / 2} \mathrm{~d} t \\
= & \frac{(\pi / 2)^{1 / 2}}{\Gamma(v+1 / 2)} 2^{v-n} \mathrm{e}^{-2 c} \int_{0}^{\infty} \mathrm{e}^{-c t} t^{v-1 / 2}(1+t / 2)^{v-1 / 2-n} \mathrm{~d} t \\
= & \frac{(\pi / 2)^{1 / 2}}{\Gamma(v+1 / 2)} 2^{v-n} \mathrm{e}^{-2 c}\left(\frac{\mathrm{d}}{\mathrm{d} c}\right)^{n} \int_{0}^{\infty} \mathrm{e}^{-c t} t^{v-1 / 2-n}\left(1+\frac{t}{2}\right)^{v-1 / 2-n} \mathrm{~d} t \\
= & \mathrm{e}^{-2 c} 2^{v-n} \frac{\Gamma(v-n+1 / 2)}{\Gamma(v+1 / 2)}\left(\frac{\mathrm{d}}{\mathrm{d} c}\right)^{n}\left[\mathrm{e}^{c} P_{v-n}(c)\right],
\end{aligned}
$$

where $P_{v}(c)=c^{-v} K_{v}(c)$. Therefore,

$$
\begin{aligned}
\frac{\mathrm{d}}{\mathrm{d} c} P_{v}(c) & =-c P_{v+1}(c), \\
\left(\frac{\mathrm{d}}{\mathrm{d} c}\right)^{i} P_{v}(c) & =\sum_{k=0}^{[i / 2]} b_{k i}(-1)^{i+k} c^{i-2 k} P_{v+i-k}(c),
\end{aligned}
$$

where $\left\{b_{k, i+1}\right\}, k=0,1, \ldots,[(i+1) / 2]$ are given in terms of $\left\{b_{k, i}\right\}_{k=0,1, \ldots,[i / 2]}$ by

$$
b_{k, i+1}=b_{k-1, i}(i-2 k+2)+b_{k, i}
$$

with the initial conditions $b_{0, i}=1$ and $b_{1,1}=0$. Hence, $b_{k i}=i ! /\left\{(i-2 k) ! 2^{k} k !\right\}$. Using these results, one can show that $R_{n}(A, x)$ satisfies (2.6).

Note 3.1. $\theta=0$ yields (4.34) of [3].

Note 3.2. In the computations we used the result on p. 5 of [5] that

$$
K_{v}(c)=\frac{\pi}{2 \sin v \pi}\left(I_{-v}-I_{v}\right),
$$

where

$$
I_{v}=\sum_{m=0}^{\infty}\left(\frac{c}{2}\right)^{2 m+v} / m ! \Gamma(m+v+1)
$$

For $c>2$ we used the modified asymptotic expansion of $K_{v}(c)$ given in Section 8.43 of [6].

Proof of Theorem 2.4. The proof is similar - but easier and uses the fact that $K_{v}(c)=K_{-v}(c)$.

Proof of Note 2.4. A fairly straightforward generalization of VI.4.5, p. 230 and VII.2.3, p. 272 of [7].

Lemma 1. Let $Q$ and $P$ denote the probability distributions corresponding to the processes $\gamma_{1}=(y+\theta p) \psi$ and $\gamma_{2}=y \psi$ for general $\psi$. Let $X=-\int_{0}^{1} \ddot{p}(u) y(u) \mathrm{d} u$. Then

$$
\begin{aligned}
\text { (i) } \quad E \gamma_{2}(t) X & =b(t) ; \\
\text { (ii) } \quad \mathrm{d} Q / \mathrm{d} P & =\exp \left[\theta x-2^{-1} \theta^{2} \int_{0}^{1} \dot{p}^{2}\right] .
\end{aligned}
$$


Proof. (i) is straightforward. (ii) follows from (i) and Problem 11 of [7], p. 124.

Proof of Theorem 2.5. By [7] (Pb. 16, p. 199),

$$
P(-\lambda \leq y(t) \leq \lambda \text { for } 0<t<v \mid y(0)=y)=K(v, 0, y, \lambda),
$$

defined in the theorem. Since $\{y(t), t \in(0, v)\}$ and $\{y(t), t \in(v, 1)\}$ are conditionally independent given $y(v)=y$,

$$
\int_{A} y(t) \mathrm{d} P=-\int_{A^{c}} y(t) \mathrm{d} P=-\int_{-\lambda}^{\lambda} y f_{t}(y, \lambda) \mathrm{d} y=0
$$

where

$$
A=\left\{f(\cdot): \sup _{(0,1)}|f(t)| \geq \lambda\right\}
$$

and

$$
\begin{gathered}
f_{v}(y, \lambda)=\phi\left(x\left(v-v^{2}\right)^{-1 / 2}\right)\left(v-v^{2}\right)^{-1 / 2} K(v, 0, y, \lambda) K(1-v, 0, y, \lambda)=f_{v}(-y, \lambda) \\
P(\sup |y+\theta p| \geq \lambda)=Q(A)=\int_{A} \frac{\mathrm{d} Q}{\mathrm{~d} P} \mathrm{~d} P
\end{gathered}
$$

which by Lemma 1 has coefficient of $\theta$ equal to $\int_{A} X \mathrm{~d} P=0$ and coefficient of $\theta^{2} / 2$ equal to

$$
\int_{A}\left(X^{2}-\int \dot{p}^{2}\right) \mathrm{d} P
$$

Now

$$
\int_{A} X^{2} \mathrm{~d} P=\int \ddot{p}(u) \mathrm{d} u \int \ddot{p}(v) \mathrm{d} v \int_{A} y(u) y(v) \mathrm{d} P .
$$

Therefore,

$$
\begin{aligned}
& \min (u, v)-u v-\int_{A} y(u) y(v) \mathrm{d} P= \\
& \int_{A^{c}} y(u) y(v) \mathrm{d} P \\
= & \int_{-\lambda}^{\lambda} x \mathrm{~d} x \int_{-\lambda}^{\lambda} z \mathrm{~d} z f_{u v}(x z \lambda) \cdot(\text { joint density of } x=y(u) \text { and } z=y(v)),
\end{aligned}
$$

where

$$
f_{u v}(x z \lambda)=P\{-\lambda \leq y(t) \leq \lambda \text { in }(0,1) \mid y(u)=x, y(v)=z\},
$$

which is a given in the theorem using conditional independence and (7), p. 199 of [7] with $\alpha=\beta=\lambda$, which implies that

$$
K(v-u, x, z, \lambda)=P\{-\lambda \leq y(t) \leq \lambda \text { in }(u, v) \mid y(u)=x, y(v)=z\} .
$$


The theorem follows using $P(A)=\alpha$ and

$$
\iint \ddot{p}(u) \ddot{p}(v)(u \wedge v-u v) \mathrm{d} u \mathrm{~d} v=\int \dot{p}^{2}-\left(\int \dot{p}\right)^{2}
$$

where $s \wedge t=\min (s, t)$.

Proof of Theorem 2.6. Let $A=\{f(\cdot): \sup f+\sup -f \geq \lambda\}$,

$$
(\sup y+\sup -y)|y(t)=x \stackrel{\mathcal{L}}{=}(\sup y+\sup -y)| y(t)=-x .
$$

It follows that

$$
\begin{aligned}
{\left[\frac{\partial}{\partial \theta} Q(A)\right]_{\theta=0} } & =\int_{A} y(t) \mathrm{d} P=-\int_{A^{c}} y(t) \mathrm{d} P=0, \\
{\left[\frac{\partial^{2}}{\partial \theta^{2}} Q(A)\right]_{\theta=0} } & =\int_{A} X^{2} \mathrm{~d} P-\int \dot{p}^{2} P(A), \\
\int_{A^{c}} y(u) y(v) \mathrm{d} P & =\int x \mathrm{~d} x \int z \mathrm{~d} z f_{u v}(x z \lambda) \\
& \times(\text { joint density of } x=y(u) \text { and } z=y(v)),
\end{aligned}
$$

where

$$
f_{u v}(x z \lambda)= \begin{cases}f_{v u}(z x \lambda), & v<u \\ P\{\sup y+\sup -y \leq \lambda \mid y(u)=x, y(v)=z\}, & v>u\end{cases}
$$

Let

$$
\begin{aligned}
& D_{1}^{+}=\sup _{(0, u)} y, D_{2}^{+} \quad=\sup _{(u, v)} y, D_{3}^{+}=\sup _{(v, 1)} y \\
& D_{1}^{-}=\sup _{(0, u)}-y, D_{2}^{-}=\sup _{(u, v)}-y, D_{3}^{-}=\sup _{(v, 1)}-y
\end{aligned}
$$

So, for $v>u$,

$$
\begin{aligned}
f_{u v}(x z \lambda) & =P\left\{D_{i}^{+}+D_{j}^{-} \leq \lambda, i, j=1,2,3 \mid y(u)=x, y(v)=z\right\} \\
& =\iiint \iiint_{\substack{x_{i}+z_{j} \leq \lambda \\
i, j=1,2,3}} \prod_{i=1}^{3} \frac{\partial^{2}}{\partial x_{i} \partial z_{i}} P\left\{D_{i}^{+} \leq x_{i}, D_{i}^{-} \leq z_{i} \mid y(u)=x, y(v)=z\right\} \mathrm{d} x_{i} \mathrm{~d} z_{i}
\end{aligned}
$$

by conditional independence. For $d=v-u$,

$$
\begin{aligned}
& P\left\{D_{2}^{+} \leq A, D_{2}^{-} \leq B \mid y(u)=x, y(v)=z\right\} \\
= & P\{-B \leq y(t) \leq A \text { for } t \in(u, v) \mid y(u)=x, y(v)=z\} \\
= & 1-\sum_{m=1}^{\infty}\left[\exp \left(-2 A_{m} / d\right)+\exp \left(-2 B_{m} / d\right)-\exp \left(-2 C_{m} / d\right)-\exp \left(-2 D_{m} / d\right)\right]
\end{aligned}
$$

by (6), p. 199 of [7] since

$$
T(s)=\mathrm{d}^{-1 / 2}\{y(u+\mathrm{d} s)-(1-s) x-s z \mid y(u)=x, y(v)=z\}
$$

is a Brownian Bridge on $[0,1]$. So,

$$
\partial^{2} /(\partial A \partial B) P\left\{D_{2}^{+} \leq A, D_{2}^{-} \leq B \mid y(u)=x, y(v)=z\right\}=H(d, x, z, A, B)
$$


of the theorem. So, $f_{u v}(x z \lambda)$ is as given in the theorem. The theorem follows.

Proof of Example 2.4. Note $p(x)=-x, x \in[0,1 / 2], p(x)=-1+x, x \in[1 / 2,1]$. For (i), we have

$$
\begin{aligned}
& 1-P\{\sup |y+\theta p|>\lambda)=P(-\lambda<y+\theta p<\lambda \in(0,1)\} \\
= & \int P\{-\lambda+\theta x<y(x)<\lambda+\theta x \in(0,1 / 2) \mid y(1 / 2)=r\} \\
& \times P\{-\lambda+\theta(1-x)<y(x)<\lambda+\theta(1-x) \in(1 / 2,1) \mid y(1 / 2)=r\} \mathrm{d} \Phi(2 r) \\
= & \int H\left(2^{1 / 2} \lambda, 2^{1 / 2}(\lambda+r-\theta / 2), 2^{1 / 2} \lambda, 2^{1 / 2}(\lambda-r+\theta / 2)\right)^{2} \mathrm{~d} \Phi(2 r)
\end{aligned}
$$

by symmetry and by (3.1), where

$$
\begin{aligned}
H(a, b, \alpha, \beta) & =P\{-a(1-t)-b t<y(t)<\alpha(1-t)+\beta t \in(0,1)\} \\
& =1-\sum_{m=1}^{\infty}\left[\exp \left(-2 A_{m}\right)+\exp \left(-2 B_{m}\right)-\exp \left(-2 C_{m}\right)-\exp \left(-2 D_{m}\right)\right]
\end{aligned}
$$

for $a, b, \alpha, \beta$ non-negative and $H(a, b, \alpha, \beta)=0$ otherwise, where $A_{m}, B_{m}, C_{m}, D_{m}$ are given in Problem 15, p. 199 of [7], (i) follows on simplification since $H(x, \mu)=H(\mu, \mu+x, \mu, \mu-x)$, (ii) follows similarly since if

$$
G(a, b)=P\{\sup (y+\theta p) \leq a, \sup -(y+\theta p) \leq b\}
$$

then the AP is

$$
1-\iint_{a+b<\lambda}\left(\partial^{2} /(\partial a \partial b)\right) G(a, b) \mathrm{d} a \mathrm{~d} b=1-\int_{0}^{\lambda} G_{2}\left(\lambda-b_{0}, b_{0}\right) \mathrm{d} b_{0}
$$

for $G_{2}(a, b)=(\partial / \partial b) G(a, b)$ so that

$$
G_{2}\left(\lambda-b_{0}, b_{0}\right) \mathrm{d} b_{0}=2^{3 / 2} \int(\partial H / \partial a+\partial H / \partial b) H \mathrm{~d} \phi(x)
$$

for $H=H(a, b, \alpha, \beta)$ evaluated at

$$
a=2^{1 / 2} b_{0}, b=2^{1 / 2}\left(b_{0}-\theta / 2\right)+x, \alpha=2^{1 / 2}\left(\lambda-b_{0}\right), \beta=2^{1 / 2}\left(\lambda-b_{0}+\theta / 2\right),
$$

since by conditioning on $y(1 / 2)=x / 2^{1 / 2}, G(a, b)$ is found to be

$$
\int H\left(2^{1 / 2} b, 2^{1 / 2} b+x-\theta / 2^{1 / 2}, 2^{1 / 2} a, 2^{1 / 2} a-x+\theta / 2^{1 / 2}\right)^{2} \mathrm{~d} \Phi\left(2^{1 / 2} x\right) .
$$

The range of integration follows since $H=0$ if $a, b, \alpha$ or $\beta$ are negative.

Acknowledgements. The authors would like to thank the Editors-in-Chief, the Associate Editor and the two referees for carefully reading the paper and for their comments which greatly improved the paper.

\section{REFERENCES}

[1] M. Abramowitz and I.A. Stegun, Handbook of Mathematical Functions with Formulas, Graphs, and Mathematical Tables. National Bureau of Standards Appl. Math. Ser. 55. U.S. Government Printing Office, Washington, D.C. (1964).

[2] J. Andel, Local asymptotic power and efficiency of tests of Kolmogorov-Smirnov type. Ann. Math. Statist. 38 (1967) 1705-1725.

[3] T.W. Anderson and D.A. Darling, Asymptotic theory of certain "goodness of fit" criteria based on stochastic processes. Ann. Math. Statist. 23 (1952) 193-212. 
[4] R. Courant and D. Hilbert, Methods of Mathematical Physics, volume I. Interscience Publishers, Inc., New York (1953).

[5] A. Erdelyi, W. Magnus, F. Oberhettinger and F.G. Tricomi, Higher Transcendental Functions, volume II. Robert E. Krieger Publishing Co., Inc., Melbourne, FL (1981).

[6] I.S. Gradshteyn and I.M. Ryzhik, Table of Integrals, Series, and Products, sixth edition. Academic Press, San Diego (2000).

[7] J. Hajek and Z. Sidak, Theory of Rank Tests. Academic Press, Inc., New York (1967).

[8] A. Janssen, Principal component decomposition of non-parametric tests. Probab. Theory Related Fields 101 (1995) 193-209.

[9] A. Janssen, Global power functions of goodness of fit tests. Ann. Statist. 28 (2000) 239-253.

[10] A. Janssen and F. Marohn, On statistical information of extreme order statistics, local extreme value alternatives, and Poisson point processes. J. Multiv. Anal. 48 (1994) 1-30.

[11] C. Jordan, Calculus of Finite Differences, third edition. Chelsea Publishing Co., New York (1965).

[12] A.N. Kolmogorov, Confidence limits for an unknown distribution function. Ann. Math. Statist. 12 (1941) 461-463.

[13] E.L. Lehmann and J.P. Romano, Testing Statistical Hypotheses, third edition. Springer, New York (2005).

[14] H. Milbrodt and H. Strasser, On the asymptotic power of the two-sides Kolmogorov-Smirnov. J. Statist. Plann. Inference 26 (1990) $1-23$.

[15] E.S. Pearson and H.O. Hartley, Biometrika Tables for Statisticians, volume II. Cambridge University Press, New York (1972).

[16] D. Quade, On the asymptotic power of the one-sample Kolmogorov-Smirnov Tests. Ann. Math. Statist. 36 (1965) $1000-1018$.

[17] J. Rahnenführer, On preferences of general two-sided tests with applications to Kolmogorov Smirnov-type tests. Statist. Decisions 21 (2003) 149-170.

[18] S. Shapiro and M. Wilk, An analysis of variance test for normality. Biometrika 52 (1965) 591-611.

[19] G.R. Shorak and J.A. Wellner, Empirical Processes with Applications to Statistics. Wiley, New York (1986).

[20] G.P. Steck, Rectangle probabilities for uniform order statistics. Ann. Math. Statist. 42 (1971) 1-11.

[21] M.A. Stephens, The goodness-of-fit statistic $V_{N}$ : Distribution and significance points. Biometrika 52 (1965) 309-321.

[22] M.A. Stephens, Tests for normality. Technical Report No. 152, November 10, 1969, Department of Statistics, Stanford University (1969).

[23] M.A. Stephens, Kolmogorov type tests for exponentiality. Technical Report No. 154, Department of Statistics, Stanford University (1970).

[24] H. Strasser, Global extrapolations of local efficiency. Statist. Decisions 8 (1990) 11-26. 